\title{
Alterations of autophagy in knee cartilage by treatment with treadmill exercise in a rat osteoarthritis model
}

\author{
XIAONING ZHANG ${ }^{1}$, YUE YANG $^{1}, \mathrm{XIN} \mathrm{LI}^{2}, \mathrm{HE} \mathrm{ZHANG}{ }^{1}, \mathrm{YI} \mathrm{GANG}^{1}$ and $\mathrm{LUNHAO} \mathrm{BAI}^{1}$ \\ ${ }^{1}$ Department of Orthopaedic Surgery, Shengjing Hospital of China Medical University, Shenyang, Liaoning 110022; \\ ${ }^{2}$ Department of Anesthesiology, Liaoning Cancer Hospital, Shenyang, Liaoning 110042, P.R. China
}

Received January 15, 2018; Accepted October 19, 2018

DOI: $10.3892 / \mathrm{ijmm} .2018 .3948$

\begin{abstract}
The aim of the present study was to investigate potential alterations in the articular cartilage in a rat model of monosodium iodoacetate (MIA)-induced osteoarthritis (OA) with or without treatment with moderate treadmill exercise. A total of 30 male Sprague-Dawley rats were randomly divided into three groups $(\mathrm{n}=10)$, including the control, $\mathrm{OA}$ and $\mathrm{OA}$ with treadmill exercise (OAE) groups. Rats were evaluated upon completing the treadmill exercise program (speed, $18 \mathrm{~m} / \mathrm{min}$; $30 \mathrm{~min} /$ day; 5 days/week for 4 weeks). Interleukin (IL)-1 $\beta$ and IL-4 levels in the serum and intra-articular lavage fluid (IALF) were measured by ELISA. Alterations in articular cartilage and synovium were also evaluated by histology, immunohistochemistry, western blotting and reverse transcription-quantitative polymerase chain reaction. The results revealed that IL-1 $\beta$ in the serum and IALF decreased in the OAE group, whereas IL-4 increased, and histological evaluation indicated that the OAE group had a clear treatment response. However, the expression of type II collagen in the articular cartilage increased in the OAE group as compared with the OA group, whereas ADAMTS5 expression decreased. In contrast to light chain 3B (LC3B), the protein expression levels of BECLIN1 and sequestosome 1 (SQSTM1) were increased in the OA group. In addition, a significant increase was observed between OA and OAE groups in LC3B and SQSTM1 protein levels, whereas no change was observed in BECLIN1 levels between the OA and OAE groups in the superficial and deep zones. The results of western blotting demonstrated that LC3II was notably decreased in the OA group and partially increased in the OAE group. The mRNA expression levels of LC3B and SQSTM1 increased in the $\mathrm{OA}$ and OAE groups, with a significant difference observed
\end{abstract}

Correspondence to: Dr Lunhao Bai, Department of Orthopaedic Surgery, Shengjing Hospital of China Medical University, 36 Sanhao Street, Heping, Shenyang, Liaoning 110022, P.R. China

E-mail: bailh@sj-hospital.org

Key words: osteoarthritis, autophagy, articular cartilage, treadmill exercise, cartilage zone between the two groups, while a concomitant decrease was detected in BECLIN1 levels. In conclusion, 30 min of treadmill exercise had an evident protective effect in the articular cartilage of rats with MIA-induced OA and may promote autophagy in the articular cartilage.

\section{Introduction}

Osteoarthritis (OA) is a chronic joint disease characterized by the progressive degradation of articular cartilage, accompanied by remodeling of the subchondral bone and consequent articular dysfunction. OA affects $20-25 \%$ of individuals aged $>45$ years and $37.4 \%$ of individuals aged $\geq 60$ years old $(1,2)$. Knee OA may cause severe pain, physical disability and reduced quality of life. Furthermore, OA is one of the major reasons for knee arthroplasty and leads to high healthcare costs $(3,4)$.

A variety of risk factors contribute to OA, including age, gender, obesity, history of knee injury, genetic factors and nutritional factors (5). Research in the field of molecular biology has identified an imbalance in the homeostasis of the cartilage matrix during OA, as well as inflammation caused by various biomarkers, such as prostaglandins, adipokines and cytokines among others (6).

Moderate physical exercise is recognized to be an effective therapeutic treatment for knee OA (7). Research revealed that a moderate level of exercise is beneficial in rat knee OA models, and affects subchondral or trabecular bone metabolism (8-10). Various cytokines, growth factors and proteins secreted by osteoclasts and osteoblasts of OA subchondral bone cysts aggravate the disease and are alleviated by moderate physical exercise (11-14).

Autophagy, as an essential cellular homeostatic mechanism in eukaryotic cells, involves continuous biosynthesis and renewal of cellular organelles and macromolecules. It is recognized that autophagy serves an important role in the knee OA process, in which it has been demonstrated that multiple signal transduction pathways may be involved (15). Previous studies reported that autophagy in chondrocytes mainly serves as a protective response of cells to negative stimulation, such as hypoxia (16), glucose starvation or nutrient insufficiency (17), aging (18) and certain inflammatory mediators (19-21). Nevertheless, complex results have been derived from in vivo studies $(18,19,22)$. Autophagy may not only be a protective 
or homeostatic mechanism in normal or OA cartilage, but may also be an essential process for the differentiation and maturation of chondrocytes, which occurs constantly during the pathological process of OA (23).

A previous study reported that physical exercise affects autophagy in numerous tissues and organs, including muscle, adipose, heart and brain tissues (24). The present study was designed to detect the alterations in autophagy in the cartilage following treadmill exercise using a rat OA model that was induced by monosodium iodoacetate (MIA) injection. The results may help to develop a novel theory regarding the treatment effect of exercise on OA.

\section{Materials and methods}

Experimental animals. The experimental protocol was approved by the Ethics Committee of Shengjing Hospital, China Medical University (Shenyang, China). A total of 30 male Sprague-Dawley rats $(230 \pm 10 \mathrm{~g}$; 8 weeks old; specific-pathogen-free) were purchased from HFK Bioscience Co., Ltd. (Beijing, China) and kept in plastic cages with sawdust bedding in a controlled environment $\left(22 \pm 2^{\circ} \mathrm{C}\right.$; $70 \%$ humidity) under a 12:12-h light/dark cycle with the lights turned on at 06:00 a.m. Animals were able to move freely in the cages, were fed a standard diet and had access to tap water ad libitum. The body weight of rats was recorded weekly prior to the treadmill exercise each Monday. Adaptive exercise was applied to all animals at a speed of $10 \mathrm{~m} / \mathrm{min}$ for $10 \mathrm{~min} /$ day prior to regular treadmill exercise to eliminate stress.

OA model and treadmill running protocols. The animals were randomly divided into three groups ( $\mathrm{n}=10$ per group), as follows: Control (sterile saline injection only), OA (MIA injection) and OA with treadmill exercise (OAE). An intra-articular injection of MIA was used in both knees following the protocol described in our previous study (25), in order to generate the OA model according to the study of Guzman et al (26). All rats were kept sedentary in the cages; however, the OAE group began treadmill exercise at $24 \mathrm{~h}$ post-injection with a speed of $18 \mathrm{~m} / \mathrm{min}$ for $30 \mathrm{~min} /$ day (moderate exercise) for 5 days/week, which is considered to be beneficial for cartilage damage caused by MIA injection. The treatment schedule is shown in Fig. 1A.

Sampling and tissue preparation. After 4 weeks of exercise, all animals were anesthetized $2 \mathrm{~h}$ subsequent to the last exercise session. Blood samples $(3 \mathrm{ml})$ were obtained immediately and centrifuged at $3,000 \mathrm{x} \mathrm{g}$ for $10 \mathrm{~min}$ in $4^{\circ} \mathrm{C}$ to obtain the serum. All rats were euthanized, and the joints of the left knee were separated and fixed in 4\% paraformaldehyde solution at room temperature. Intra-articular lavage fluid (IALF) was obtained from the synovial cavity of the right knee by injection and recovery with $0.2 \mathrm{ml}$ PBS three times using a 1-ml syringe. Cartilage was scraped off from the weight-bearing area of the condyles of the right femur and tibia using a scalpel, and was carefully collected. The left knee joints were kept at room temperature, while other samples were kept at $-80^{\circ} \mathrm{C}$.

Histological analysis. Following 2 weeks of soaking in $4 \%$ paraformaldehyde solution, the left knee joints were washed with PBS five times (10 min/wash) and transferred to a $10 \%$ EDTA solution (EDTA-2Na, dissolved in 0.1 M PBS) to decalcify the samples. Subsequently, samples were kept at a constant temperature of $37^{\circ} \mathrm{C}$ for 1 month and the solution was renewed every 3 days. Dehydration in an ethanol series and embedding in paraffin was performed successively following the decalcification, and serial 4.5- $\mu \mathrm{m}$ sagittal sections were then cut for histological examination. Hematoxylin and eosin (H\&E) staining and toluidine blue staining were used to obtain scores according to the Osteoarthritis Research Society International (OARSI) and the modified Mankin scoring systems, respectively, in order to grade and stage OA development in cartilage (27). Synovitis scoring, which reflects the inflammation state in the synovium (28), was also performed by $H \& E$ staining.

ELISA of IALF and serum levels. IL-1 $\beta$ and IL-4 levels in the knee IALF and serum were detected using ELISA kits (cat. nos. ml037361 and ml102825; Shanghai Enzyme-linked Biotechnology Co., Ltd., Shanghai, China), following the manufacturer's protocol. Additionally, the concentration of protein in the IALF was measured using a bicinchoninic acid (BCA) assay kit (cat. no. P0010S; Beyotime Institute of Biotechnology, Shanghai, China) to correct the error in harvesting.

Immunohistochemical assay. Left knee joint sections were deparaffinized in xylene and rehydrated in graded ethanol and water. Enzymatic antigen retrieval (cat. no. AR0026; Boster Biological Technology, Pleasanton, CA, USA) was performed for $30 \mathrm{~min}$ at $37^{\circ} \mathrm{C}$ for antigen retrieval, and left knee joint sections were then stained using a two-step method, according to the protocol of the SPlink Detection kit (cat. no. SP-9001; Zhongshan Golden Bridge Biotechnology Co., Ltd., Beijing, China). The sections were incubated overnight at $4^{\circ} \mathrm{C}$ with rabbit polyclonal antibodies against type II collagen (1:500 dilution; cat. no. ab34712), light chain 3 3 (LC3B; 1:100 dilution; cat. no. ab48394), BECLIN1 (1:800 dilution; cat. no. ab62557) (all from Abcam, Cambridge, MA, USA) and sequestosome 1 (SQSTM1; 1:20 dilution; cat. no. 8420-1-AP; ProteinTech Group, Wuhan, China). Sections were then incubated with biotinylated secondary antibody and streptavidin/horseradish peroxidase were incubated for $30 \mathrm{~min}$ at room temperature successively according to the instructions of the SPlink Detection kit. Finally, the sections were visualized with 3,3-diaminobenzidine tetrahydrochloride for $1 \mathrm{~min}$ and counterstained with hematoxylin for $5 \mathrm{~min}$. The negative control group was assayed according to the abovementioned procedure, by substituting the primary antibody with PBS. The type II collagen content was evaluated based on the optical density of positive area measured using the image analysis software Image-Pro Plus, version 6.0 (Media Cybernetics, Inc., Rockville, MD, USA). To evaluate the positive cells of LC3B, BECLIN1 and SQSTM1 in the different zones of the cartilage (29), three images of each protein were captured under x200 magnification, representing the cartilages in the loaded parts. The total number and positive number of chondrocytes, as well as the number of different zones, were counted in each image, respectively. Finally, the percentage of positive cells was calculated. 


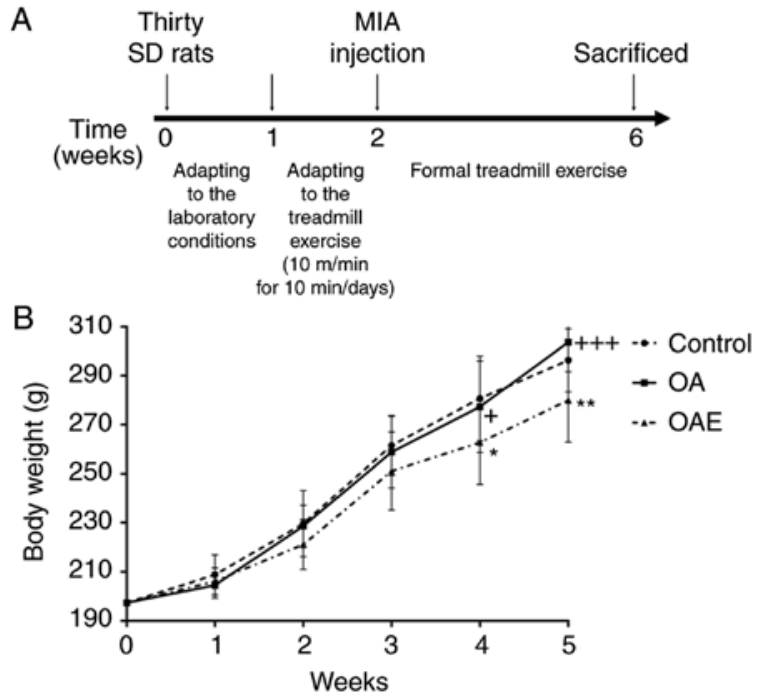

Figure 1. (A) Treatment schedule for the rats. (B) Body weight during the treadmill exercise. All rats received a standard diet, and body weights were measured weekly. No significant difference was detected between the control and OA group during exercise. Differences between control and OAE, OA and OAE groups in weeks 4 and 5 were significant. ${ }^{*} \mathrm{P}<0.05$ and ${ }^{* *} \mathrm{P}<0.01$ vs. control group; ${ }^{+} \mathrm{P}<0.05$ and ${ }^{+++} \mathrm{P}<0.001$ vs. OAE group. Results are expressed as the mean and $95 \%$ confidence interval (one-way analysis of variance; $n=10$ rats/group). MIA, monosodium iodoacetate; OA, osteoarthritis; OAE, OA with treadmill exercise.

Western blotting. Following removal from storage at $-80^{\circ} \mathrm{C}$, the cartilage was washed in PBS twice, and ultrasonication was performed in radioimmunoprecipitation assay lysis buffer (cat. no. P0013C) with $1 \mathrm{mM}$ phenylmethylsulfonyl fluoride (cat. no. ST506) (both from Beyotime Institute of Biotechnology). The lysates were centrifuged at $14,000 \mathrm{x}$ g for $5 \mathrm{~min}$ at $4^{\circ} \mathrm{C}$, and the supernatants were obtained to measure the protein concentration with the BCA assay kit. Equal amounts of protein $(50 \mu \mathrm{g})$ were added to an SDS-PAGE gel [8\% for type II collagen and a disintegrin and metalloproteinase with thrombospondin motifs 5 (ADAMTS5); 12\% for LC3B and $\beta$-actin] following heating with loading buffer (cat. no. NP0007; Thermo Fisher Scientific, Inc., Waltham, MA, USA) at $100^{\circ} \mathrm{C}$ for $5 \mathrm{~min}$, and then samples were transferred to polyvinylidene difluoride membranes. Following blocking at room temperature for $2 \mathrm{~h}$ with $1 \%$ bovine serum albumin (cat. no. A8020; Solarbio Science \& Technology Co., Ltd., Beijing, China) in Tris-buffered saline with $0.1 \%$ Tween-20 (TBST), the membranes were incubated with primary antibodies overnight at $4^{\circ} \mathrm{C}$, including the following: Rabbit polyclonal anti-type II collagen (molecular weight, $142 \mathrm{kDa}$; 1:5,000 dilution; cat. no. ab34712; Abcam), rabbit polyclonal anti-ADAMTS5 (molecular weight, $100 \mathrm{kDa}$; 1:200 dilution; cat. no. BA3020; Boster Biological Technology), rabbit polyclonal anti-LC3B (molecular weight, $15 \mathrm{kDa}$ for LC3-II and 17 kDa for LC3-I; 1:1,000 dilution; cat. no. ab48394; Abcam) and mouse monoclonal anti- $\beta$-actin (molecular weight, $42 \mathrm{kDa}$; cat. no. AA128; 1:1,000; Beyotime Institute of Biotechnology) antibodies. Following washing five times with TBST for 5 min each time, the peroxidase-conjugated AffiniPure goat anti-rabbit and anti-mouse $\mathrm{IgG}(\mathrm{H}+\mathrm{L})$ secondary antibodies (cat. nos. ZB5301 and ZB5305; Zhongshan Golden Bridge Biotechnology Co., Ltd.) were applied at a dilution of 1:10,000 in TBST and incubated at room temperature for
$1.5 \mathrm{~h}$. The membrane was washed with TBST again, detected with enhanced chemiluminescence (cat. no. P0018; Beyotime Institute of Biotechnology) and quantified using Image-Pro Plus software, version 6.0 (Media Cybernetics, Inc.). $\beta$-actin served as the internal reference.

Reverse transcription-quantitative polymerase chain reaction $(R T-q P C R)$. Following removal of the cartilage from the $-80^{\circ} \mathrm{C}$ freezer, a mortar and pestle were used to grind up the samples in a low-temperature environment maintained by liquid nitrogen. Approximately $50 \mathrm{mg}$ cartilage samples was collected for total RNA isolation with RNAiso Plus (cat. no. 9108; Takara Biotechnology Co., Ltd., Dalian, China) and High-Salt Solution for Precipitation (Plant) (cat. no. 9193; Takara Biotechnology Co., Ltd.). A total of $1 \mu \mathrm{g}$ total RNA was then reverse-transcribed into first-strand complementary DNA (cDNA) with a PrimeScript RT reagent kit with gDNA Eraser (cat. no. RR047A; Takara Biotechnology Co., Ltd.). The cDNA samples were used to conduct qPCR with an ABI Prism 7500 Fast Real-Time PCR system (Applied Biosystems; Thermo Fisher Scientific, Inc.) in a $20-\mu 1$ system using SYBR Premix Ex Taq II (cat. no. RR820A; Takara Biotechnology Co., Ltd.) with a two-step method $\left(95^{\circ} \mathrm{C}\right.$ for $30 \mathrm{sec}, 95^{\circ} \mathrm{C} 5 \mathrm{sec}, 40$ cycles of $60^{\circ} \mathrm{C}$ for $34 \mathrm{sec}$ ). All kits and reagents were used according to the manufacturer's protocol. The following primers were used: BECLIN1 forward, 5'-CTGGACCGAGTGACCATT CA-3' and reverse, 5'-AGACACCATCCTGGCGAGTT-3'; LC3B forward, 5'-TTCTTCCTCCTGGTGAAT GG-3' and reverse, 5'-CTGGGAGGCATAGACCATGT-3'; SQSTM1 forward, 5'-CTGTGGTGGGAACTCGCTAT-3' and reverse, 5'-GAAAGATGAGCTTGCTGTGCT-3'; $\beta$-actin forward, 5'-CACCCGCGAGTACAACCTTC-3' and reverse, 5'-CCC ATACCCACCATCACACC-3'. The expression levels of the PCR products were calculated using the $2^{-\Delta \Delta \mathrm{Cq}}$ method, with $\beta$-actin serving as the housekeeping gene (30).

Statistical analysis. All data are expressed as the mean and 95\% confidence interval (CI), and were analyzed using IBM SPSS statistical software version 20 (IBM Corp., Armonk, NY, USA). To evaluate the normality of the results, the Kolmogorov-Smirnov and Shapiro-Wilk tests (when sample size was $\leq 5$ ) were performed. Comparisons among groups were determined by one-way analysis of variance with Tukey's tests for pairwise comparisons, following the Levene's test to examine homogeneity of variance. If homogeneity of variance was missing, Games-Howell method was used as a replacement. $\mathrm{P}<0.05$ was considered to indicate a statistically significant difference.

\section{Results}

Weight change. There were no significant changes between the control and the OA group throughout the procedure. At the same time, there was a significant difference between the OAE and the control, the OAE and the OA group at the fourth and fifth week (Fig. 1B).

Histological analysis of knee cartilage. The joint cartilage of the control group exhibited a slight degeneration in the superficial zone (SZ), including cartilage matrix edema 
A

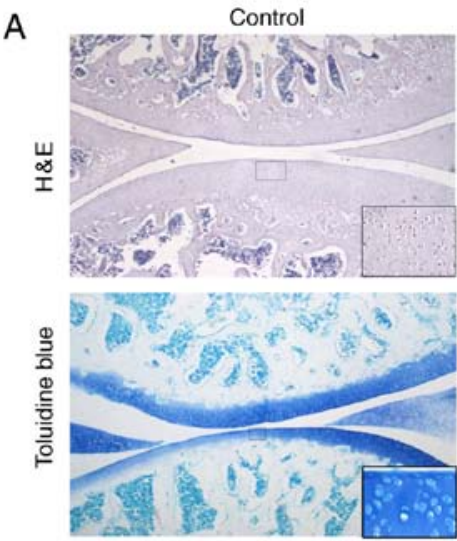

B

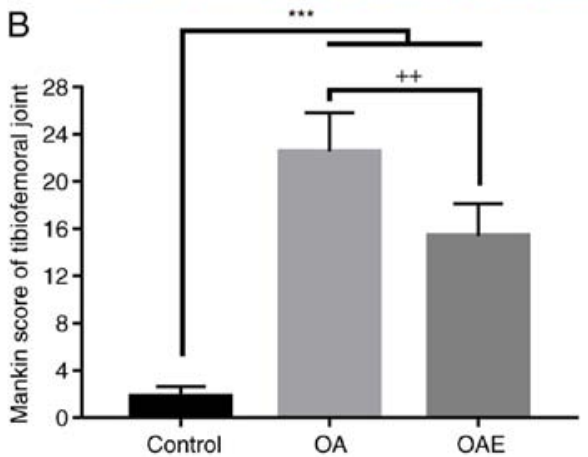

$\mathrm{OA}$
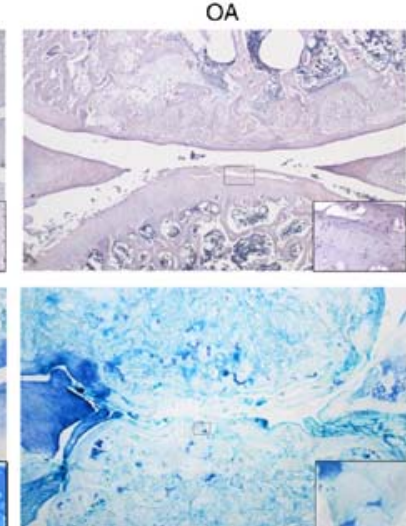

C

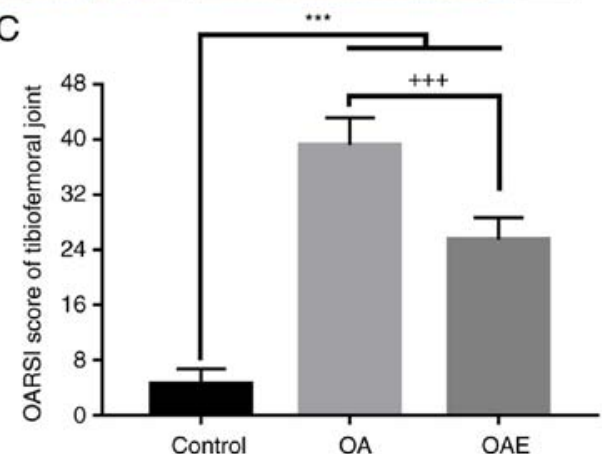

Figure 2. Histological staining and evaluation of the tibiofemoral joint. (A) H\&E and toluidine blue staining of sections in the sagittal plane. Images were captured at x40 magnification, and local magnification of a selected square was used to observe the details for each group (x200 for H\&E and x400 for toluidine blue). (B) Mankin score and (C) OARSI score for the tibiofemoral joint. Differences between the control vs. OA, control vs. OAE, and OA vs. OAE groups were statistically significant. ${ }^{* * *} \mathrm{P}<0.001,{ }^{++} \mathrm{P}<0.01$ and ${ }^{+++} \mathrm{P}<0.001$ vs. OA group. Results are expressed as the mean and $95 \%$ confidence interval (one-way analysis of variance; $n=10$ rats/group). OA, osteoarthritis; OAE, OA with treadmill exercise; H\&E, hematoxylin and eosin.

and chondrocyte proliferation (Fig. 2A). By contrast, in the OA group, severe damage was observed in sections stained with $H \& E$ and toluidine blue, such as denudation in the deep zone (DZ) and microfractures in the fibrocartilage. In the OAE group, the cartilage exhibited different alterations, from superficial fibrillation to matrix loss in the mid zone. Furthermore, the Mankin score in the control group was 1.80 (95\% CI, 0.95-2.65), while it was 22.55 (95\% CI, 19.27-25.83) in the OA group and 15.40 (95\% CI, 12.69-18.11) in the OAE group (Fig. 2B). The OARSI score was 4.55 (95\% CI, 2.36-6.74) in the control group, 39.20 (95\% CI, 35.23-43.17) in the OA group, and 25.50 (95\% CI, 22.36-28.64) in the OAE group (Fig. 2C). The OA and OAE group scores were significantly increased compared with the control, while there was a marked reduction in the OAE group in comparison with the scores in the OA group.

Type II collagen and ADAMTS5 alterations. As demonstrated via immunohistochemical analysis, type II collagen expression in the OA group was much weaker in comparison with that in the control group, and this reduction was notably attenuated by exercise in the OAE group (relative percentages, 43 and $69 \%$ in the OA and OAE groups, respectively; Fig. 3A and B). The expression of type II collagen detected via western blotting exhibited a similar trend to the immunohistochemistry results [control group, 1.87 (95\% CI, 1.28-2.46); OA group, 0.30 (95\% CI, 0.05-0.55); and OAE group, 0.97 (95\% CI, $0.35-1.58$ ); Fig. 3C and D]. On the contrary, the expression of ADAMTS5 was markedly increased in the OA group and moderately increased in the OAE group [control group, 0.16 (95\% CI, -0.07-0.38); OA group, 1.12 (95\% CI, 0.83-1.42), and OAE group, 0.47 (95\% CI, 0.08-0.87; Fig. 3C and D].

Inflammation in synovium, and $I L-1 \beta$ and $I L-4$ levels detected by ELISA. As compared with the control group, H\&E staining of the synovium of the OA group displayed an enlargement of the synovial lining cell layer and inflammatory cell infiltration, accompanied by an increase of the cellularity (Fig. 4A). However, the synovial stroma was mostly normal in the OAE group. The synovitis score in the control group was 0.90 (95\% CI, 0.22-1.58), while the scores in the OA and OAE groups were 3.20 (95\% CI, 2.49-3.91) and 2.00 (95\% CI, 1.12-2.88), respectively (Fig. 4A and B).

ELISA revealed that, compared with the control group, the IL-1 $\beta$ level was significantly increased in the serum and IALF of the OA group, whereas the expression of IL-4 was notably diminished. In the OAE group, the variation in IL-1 $\beta$ and IL-4 levels was alleviated compared with that in the OA group, although the differences between the two groups were not significant (Fig. 4C). More specifically, the serum IL-1 $\beta$ levels were $23.18(95 \% \mathrm{CI}$, $19.77-26.58 \mathrm{pg} / \mathrm{ml}), 33.75$ (95\% CI, 28.83-38.66 pg/ml) and 27.56 (95\% CI, 23.10-32.01 pg/ml) in the control,OA and OAE groups, respectively. The IL-1 $\beta$ levels in IALF were $62.31(95 \% \mathrm{CI}$, 56.71-67.91 pg/mg protein), 97.48 (95\% CI, 87.26-107.69 pg/mg protein) and 87.18 (95\% CI, 78.05-96.31 pg/mg protein) in the three groups, respectively. Furthermore, the IL-4 levels in the control, OA and OAE groups were 83.98 (95\% CI, 

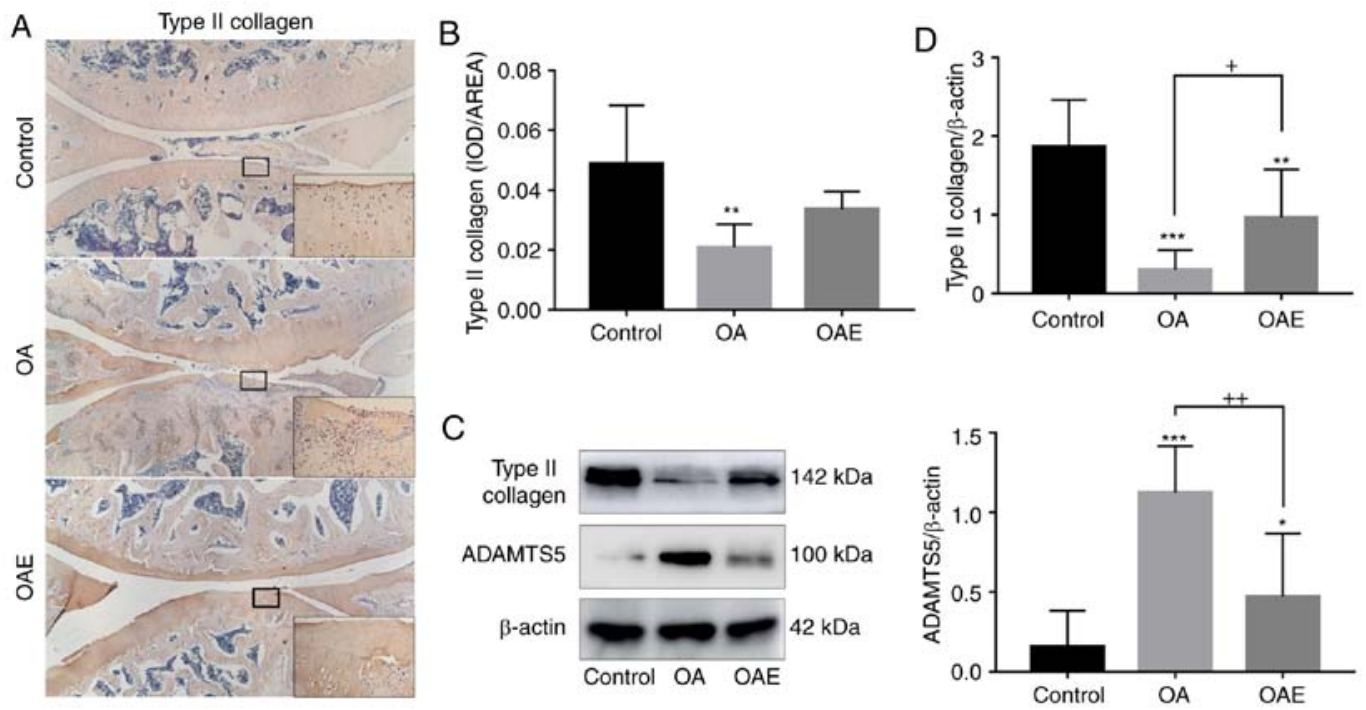

Figure 3. Type II collagen and ADAMTS5 alterations among the groups. (A) Immunohistochemical staining images and (B) quantified levels of type II collagen. Images were captured at $\times 40$ magnification, and local magnification of a selected square at $\mathrm{X} 200 \mathrm{was}$ applied. The difference between the control and OA groups was significant $(n=5)$. (C) Western blot analyses and (D) quantified results of the protein levels of type II collagen and ADAMTS5, with $\beta$-actin serving as the internal reference. For type II collagen and ADAMTS5, differences between the control vs. OA, control vs. OAE, and OA vs. OAE groups were significant $(\mathrm{n}=3)$. Results are expressed as the mean and $95 \%$ confidence interval (one-way analysis of variance). ${ }^{*} \mathrm{P}<0.05,{ }^{* *} \mathrm{P}<0.01$ and ${ }^{* * * *} \mathrm{P}<0.001$ vs. control group; ${ }^{+} \mathrm{P}<0.05$ and ${ }^{++} \mathrm{P}<0.01$. OA, osteoarthritis; OAE, OA with treadmill exercise; ADAMTS5, a disintegrin and metalloproteinase with thrombospondin motifs 5.

A
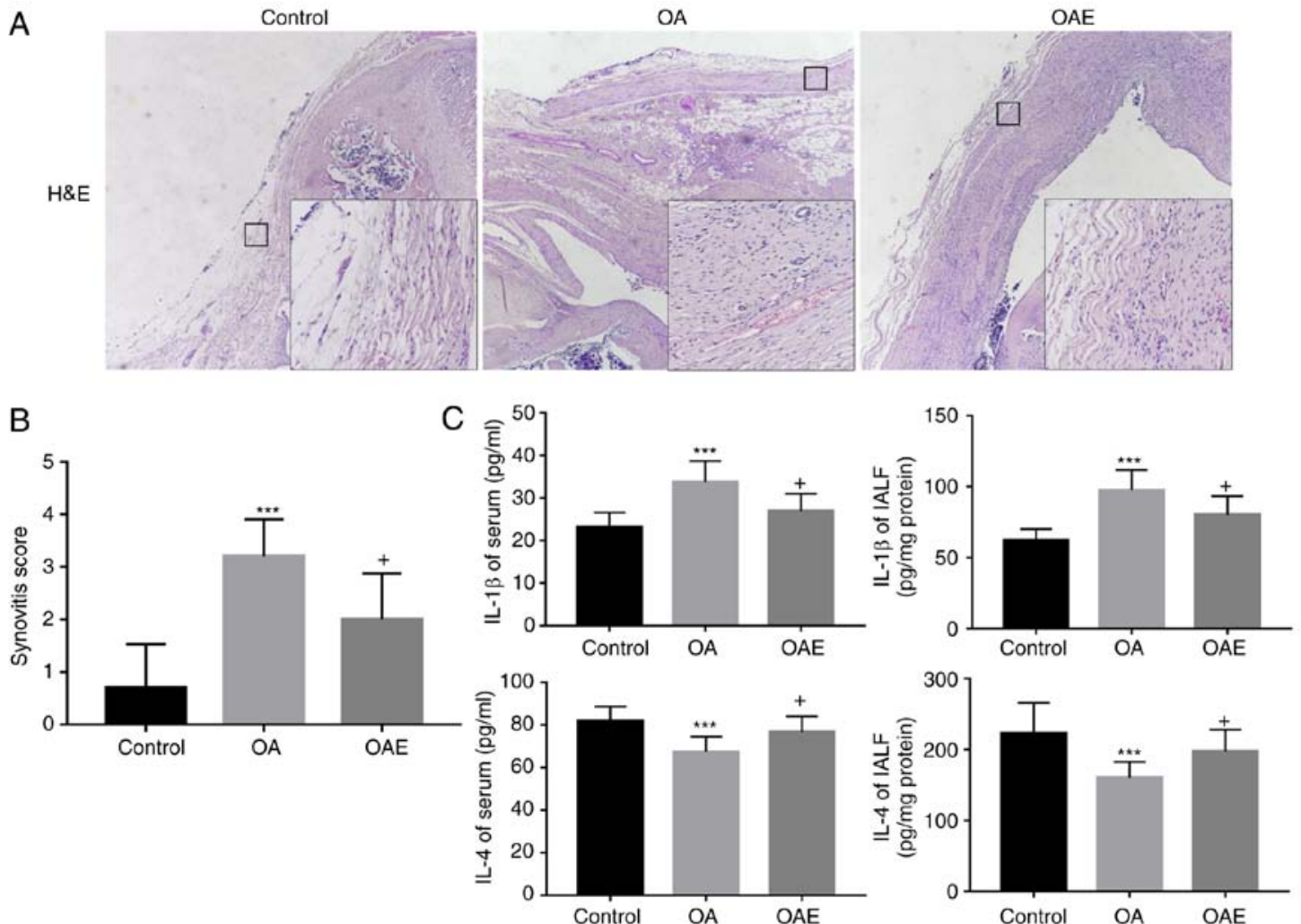

Figure 4. (A) H\&E staining for histological evaluation in synovium, and (B) synovitis score. Images were captured at x40 magnification, and local magnification of a selected square at $\times 200$ was applied. Differences in synovitis score in the control vs. OA, control vs. OAE and OA vs. OAE groups were statistically significant (n=5). (C) ELISA of IL-1 $\beta$ and IL-4 levels in the serum and IALF. Treadmill exercise reduced the increase in IL-1 $\beta$ and reversed the decrease in IL-4 caused by monosodium iodoacetate injection, in the serum and IALF ( $\mathrm{n}=10)$. Results are expressed as the mean and 95\% confidence interval (one-way analysis of variance). ${ }^{+} \mathrm{P}<0.05$ vs. OA group; ${ }^{* * * *} \mathrm{P}<0.001$ vs. control group. OA, osteoarthritis; OAE, OA with treadmill exercise; H\&E, hematoxylin and eosin; IALF, intra-articular lavage fluid; IL, interleukin.

$76.33-91.63 \mathrm{pg} / \mathrm{ml}), 67.35(95 \% \mathrm{CI}, 62.21-72.49 \mathrm{pg} / \mathrm{ml})$ and 74.75 (95\% CI, 69.02-80.48 pg/ml) in the serum, respectively; these levels in the IALF were 206.40 (95\% CI, 174.82-237.98 pg/mg protein), 160.34 (95\% CI, $144.32-176.36 \mathrm{pg} / \mathrm{mg}$ protein) and 172.63 (95\% CI, $144.76-200.50 \mathrm{pg} / \mathrm{mg}$ protein), respectively (Fig. 4C). 

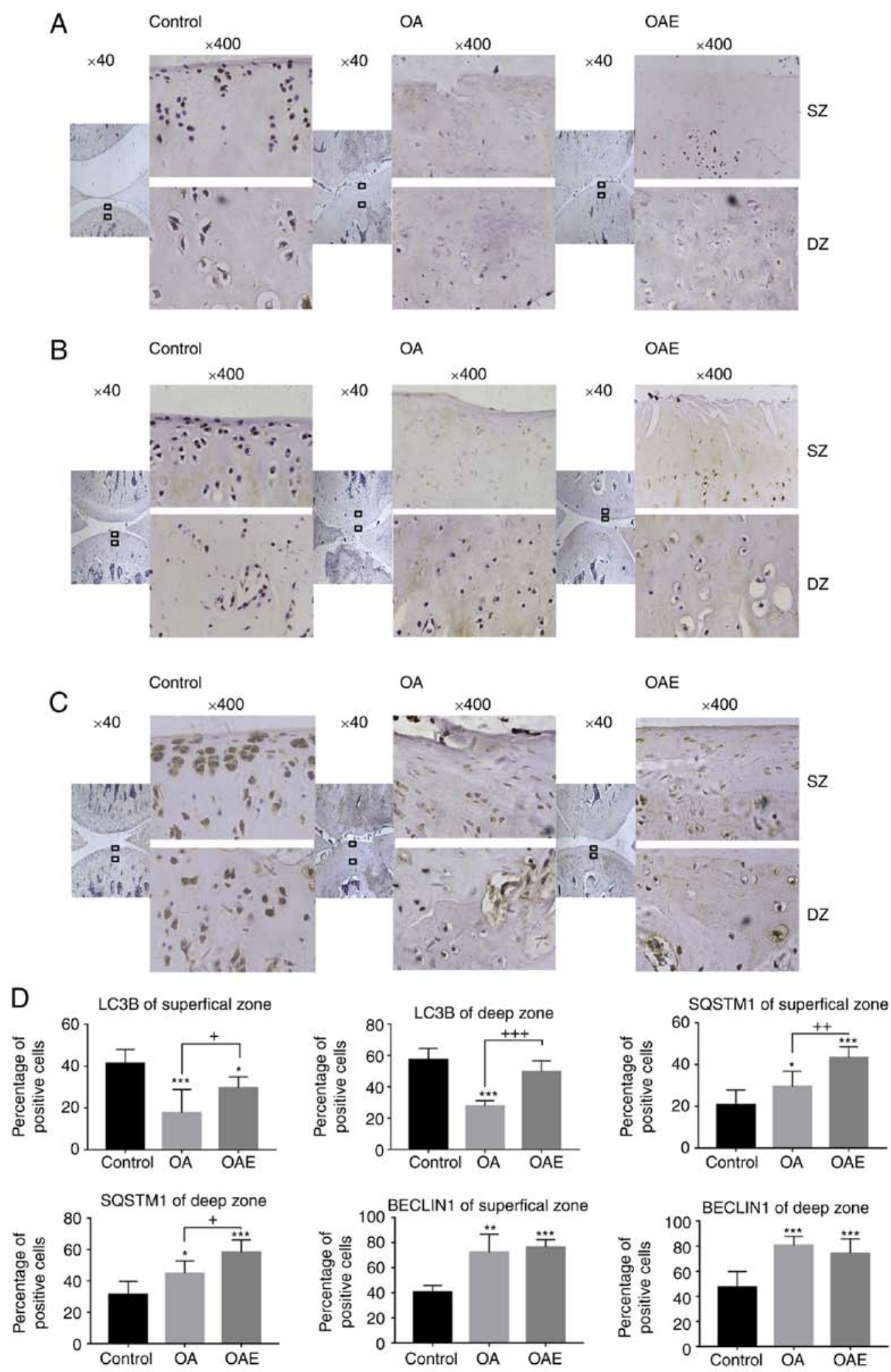

Figure 5. Immunohistochemical staining for LC3B, SQSTM1 and BECLIN1. (A) LC3B exhibited a decrease in the SZ and DZ of the cartilage in both the OA and OAE groups, while treadmill exercise increased LC3B expression in OA rats. (B) SQSTM1 expression was increased in the OA and OAE groups, while treadmill exercise evidently increased SQSTM1 level in the SZ and DZ of the cartilage. (C) BECLIN1 expression was increased in the OA and OAE groups in both the SZ and DZ of the cartilage, with no significant difference between the OA and OAE groups. (D) Results are expressed as the mean and 95\% confidence interval (one-way analysis of variance; $n=5$ ). ${ }^{*} \mathrm{P}<0.05,{ }^{* * *} \mathrm{P}<0.01$ and ${ }^{* * *} \mathrm{P}<0.001$ vs. control group; ${ }^{+} \mathrm{P}<0.05,{ }^{++} \mathrm{P}<0.01$ and ${ }^{+++} \mathrm{P}<0.001$. OA, osteoarthritis; OAE, OA with treadmill exercise; LC3B, light chain 3B; SQSTM1, sequestosome 1; SZ, superficial zone; DZ, deep zone.

Autophagy markers in the articular cartilage. As demonstrated by immunohistochemical assay, LC3B was expressed widely in the $\mathrm{SZ}$ and $\mathrm{DZ}$ of normal cartilage, with a positive detection rate of 41 and $57 \%$ of cells, respectively (Fig. 5). However, LC3B level in the OA group was markedly reduced to 18 and $26 \%$ in the SZ and DZ, respectively. In the OAE group, these values were 30 and $50 \%$, respectively. In contrast to LC3B levels, the number of SQSTM1-positive cells gradually increased in the 
A

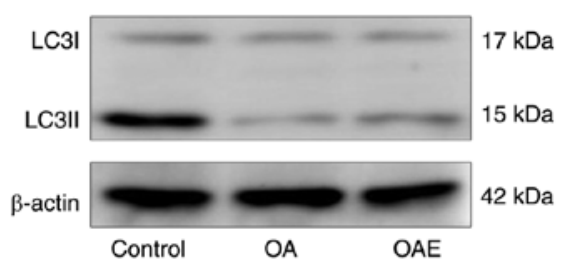

B

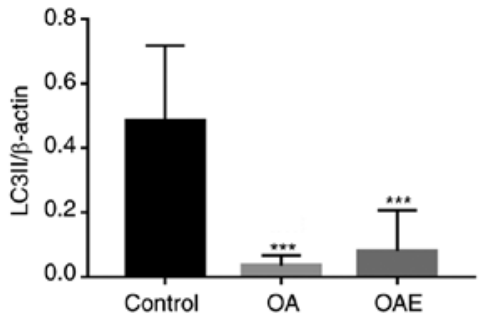

C
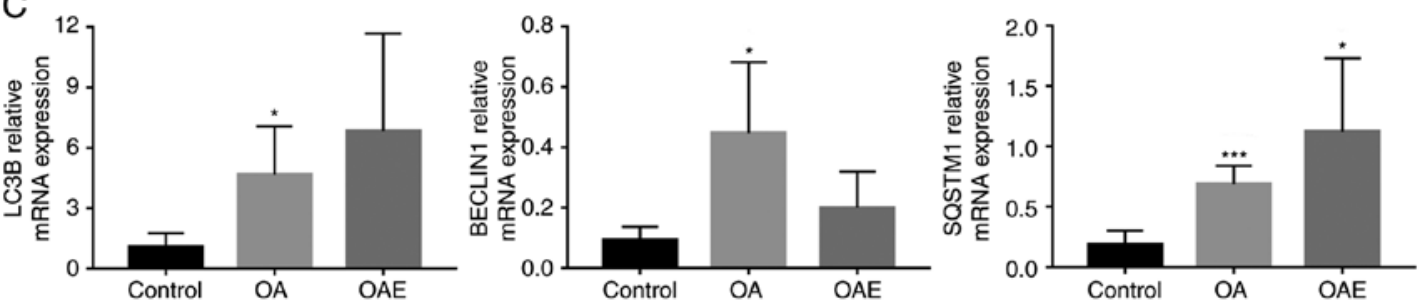

Figure 6. Western blot analysis of LC3B protein level, and reverse transcription-quantitative polymerase chain reaction of LC3B, SQSTM1 and BECLIN1 mRNA expression levels. (A) Western blot analyses and (B) quantified levels of LC3B protein, illustrating a marked decrease in the OA and OAE group compared to the control $(\mathrm{n}=3)$. No significant difference was detected between the OA and OAE groups. (C) Relative mRNA expression levels of LC3B, BECLIN1 and SQSTM1 $(n=4)$. There was no significant difference between the OA and OAE groups. Results are expressed as the mean and 95\% confidence interval (one-way analysis of variance). ${ }^{*} \mathrm{P}<0.05$ and ${ }^{* * * *} \mathrm{P}<0.001$ vs. control group. OA, osteoarthritis; OAE, OA with treadmill exercise; LC3B, light chain $3 \mathrm{~B}$; SQSTM1, sequestosome 1 .

control, OA and OAE groups, with 20,29 and $43 \%$ of cells detected respectively in the SZ, and 31, 45 and $58 \%$ detected respectively in the DZ. In terms of BECLIN1, it was notably increased in the different zones in both the OA and OAE groups compared with the control group. The positive detection rates of BECLIN1 were 41,72 and $76 \%$ in the SZ, and 47,80 and $74 \%$ in the DZ in the control, OA and OAE groups, respectively (Fig. 5).

The western blot analyses of LC3B protein are presented in Fig. 6A, while the quantitative analysis of LC3II levels among the groups is presented in Fig. 6B. A marked decrease in LC3II levels was observed in both the OA and OAE groups, with a ratio relative to the control group of 0.073 and 0.167 , respectively. RT-qPCR analysis also indicated that, compared with the control group, the mRNA expression levels were significantly increased in the OA group of LC3B and BECLIN1 and in the OA and OAE groups of SQSTM1 (Fig. 6C). Although the difference of LC3B, BECLIN1 and SQSTM1 between the OA and OAE groups was not statistically significant, the mRNA expression levels of LC3B and SQSTM1 exhibited an increasing tendency in OAE. In contrast to LC3B and SQSTM1, the mRNA expression of BECLIN1 was markedly decreased in the OAE group compared with the OA group. The relative mRNA expression of LC3B in the control, OA and OAE groups was 1.08 (95\% CI, 0.40-1.76), 4.67 (95\% CI, 2.27-7.07) and 6.83 (95\% CI, 1.99-11.68), respectively. The relative BECLIN1 levels in these groups were 0.093 (95\% CI, 0.049-1.137), 0.447 (95\% CI, 0.213-0.682) and 0.2 (95\% CI, 0.081-0.319), respectively. Similarly, the relative SQSTM1 levels were 0.188 (95\% CI, 0.074-0.302), 0.689 (95\% CI, 0.539-0.840) and 1.122 (95\% CI, 0.515-1.729), respectively (Fig. 6C).

\section{Discussion}

A number of studies have illustrated the therapeutic effect of moderate treadmill exercise in different models of OA (9-13). It has also been reported that systemic factors, such as body weight, and local factors, including muscle strength, are associated with the occurrence and development of OA (31). In the present study, it was observed that the weight gain in the OAE group was significantly lower compared with the other two groups (Fig. 1B). However, excessive exercise can promote the occurrence of OA (32), suggesting that these prophylactic treatments for $\mathrm{OA}$ are limited.

In the present study, an OA model induced by MIA injection was established, with the typical pathological alterations observed in histological analysis, while moderate treadmill exercise was applied in the OAE group and the condition has improved significantly. Alterations in cartilaginous damage were represented by the degradation of type II collagen, the major form of collagen in the matrix. Type II collagen, which is synthesized by chondrocytes and secreted to the extracellular matrix, may be degraded by various proteases, including ADAMTS5 (33). The synthesis of type II collagen is inhibited by IL-1 $\beta$ and the expression of ADAMTS5 is activated simultaneously. In the present study, the histological appearance of the synovium exhibited severe inflammation in OA group which was partially relieved in OAE group. IL-1 $\beta$, a traditional inflammatory factor, was significantly increased in the OA group, while its expression was attenuated in the OAE group, in both the serum and IALF. It was also observed that the level of IL-4, an anti-inflammatory cytokine that inhibits the proinflammatory effect of IL- $1 \beta$, was inversely correlated with that of IL-1 $\beta$. These proteins have been reported to have an important association with the occurrence of OA $(34,35)$. Compared with our previous study (25), the results of the present study indicated a consistent trend in the expression levels of IL-1 $\beta$ and type II collagen.

Autophagy, which is highly conserved in eukaryotes, has been demonstrated to serve an important role in various systems and relevant diseases, including OA. It has been reported that increased expression of LC3B and BECLIN1 mRNA results in increased autophagy in OA chondrocytes and cartilage at the outset, which represents a compensatory response to stress (22). However, in cartilage with mild or severe OA, these two proteins 
are strongly expressed in the OA cell clusters, as well as the middle zone and DZ. Their expression is however reduced in the SZ of the cartilage, and this is accompanied by an increase in cell death and cartilage damage $(36,37)$. To the best of our knowledge, studies on the alteration in autophagy in an OA model following treatment with exercise are lacking in the literature.

In the present study, the results appeared to be consistent with the findings of a previous study demonstrating that LC3B was decreased in different zones of the cartilage in OA (18). However, BECLIN1 was strongly expressed in the OA group in the SZ and DZ, and the increase was statistically significant; these results were partly in accordance with those obtained by Sasaki et al (22). This may be explained by the complexity of autophagy in different periods and zones of OA, and the distinction between a rat model induced via MIA injection and spontaneous OA in the elderly. SQSTM1 is reported to be a link between LC3 and ubiquitinated substrates (38), and the present study observed an increase in its expression in OA cartilage. A possible explanation for this effect is that the upregulated transcriptional level supplemented the consumed protein during degradation in the lysosome (39). Another important finding of the current study was that exercise markedly promoted the expression of LC3B and SQSTM1 in whole cartilage with immunohistochemical assay compared with the OA group. Notably, no differences were observed in BECLIN1 expression between the OA and OAE groups, which may be partly explained by the excessive expression in OA cartilage. In addition, western blot analysis revealed that LC3II, which serves as a marker of autophagosome formation, was markedly decreased in the OA group and this was partially alleviated in the OAE group. It was also demonstrated that, although the mRNA expression levels of the three genes of interest was elevated following prolonged stimulation with MIA, only BECLIN1 and SQSTM1 protein levels were increased in cartilage, while LC3B protein was decreased. Furthermore, the decrease detected in BECLIN1 mRNA expression in OA following treadmill exercise may be due to the complex role of BECLIN1 in autophagy and apoptosis (40). It can be hypothesized that the excessive consumption of LC3B limited the speed of autophagy and caused the accumulation of BECLIN1 and SQSTM1 proteins in the OA group, which may explain the upregulated mRNA expression of LC3B and simultaneous decrease of its protein expression. Finally, the results demonstrated that the mRNA expression levels of LC3B and SQSTM1 exhibited an increasing trend in the OAE group in comparison with the OA group, although this result was not found to be significant, likely due to the small sample size.

Our previous study demonstrated that multiple sessions of short-term exercise were associated with an improved chondroprotective effect compared with one long session of exercise for the same total time (25). The important role of autophagy has been recently reported in chondrogenesis in the regulation of the growth plate (41). In addition, a previous study indicated that the proliferation of chondrocytes was associated with mechanical stimulation (42). Therefore, it can be hypothesized that the increased expression of autophagy-associated proteins in cartilage caused by moderate treadmill exercise is associated with chondrocyte differentiation and proliferation. The present study may provide a basis for a new therapeutic strategy for OA via moderate exercise. Future studies on this topic are recommended, as several questions remain unanswered at present.

Although the effects of exercise on autophagy have been examined in numerous organs and systems, there is little evidence for bone and cartilage, particularly in OA. The findings of the present study, while preliminary, suggest that moderate exercise has a marked effect on autophagy in the cartilage in $\mathrm{OA}$, although the limitations of the detection method for autophagy caused in animal models should be considered. Furthermore, the limitations of small sample size and bias during observation cannot be neglected.

In conclusion, the present study provided strong evidence on the hypothesis that moderate treadmill exercise has a protective effect on the articular cartilage of rats with OA induced via MIA injection. In spite of several limitations, the present study suggested that moderate treadmill exercise may promote autophagy in all zones of the articular cartilage in rats with MIA-induced OA.

\section{Acknowledgements}

Not applicable.

\section{Funding}

This study was supported by the National Natural Science Foundation of China (grant no. 81772420).

\section{Availability of data and material}

The datasets used and/or analyzed during the current study are available from the corresponding author on reasonable request.

\section{Authors' contributions}

XZ, HZ and LB conceived and designed the study. XZ, YG, YY and XL conducted the experiments. HZ, YG and XL performed the statistical analysis, and designed the figures. $\mathrm{XZ}$ wrote the manuscript. YY and LB reviewed and edited the manuscript. All authors read and approved the final manuscript.

\section{Ethics approval and consent to participate}

The present study was approved by the Medical Research and New Technology Ethics Committee of Shengjing Hospital, China Medical University (Shenyang, China).

\section{Patient consent for publication}

Not applicable.

\section{Competing interests}

The authors declare that they have no competing interests.

\section{References}

1. Vaughan MW, LaValley MP, Felson DT, Orsmond GI, Niu J, Lewis CE, Segal NA, Nevitt MC and Keysor JJ: Affect and incident participation restriction in adults with knee osteoarthritis. Arthritis Care Res (Hoboken) 70: 542-549, 2018. 
2. Gómez R, Villalvilla A, Largo R, Gualillo O and HerreroBeaumont G: TLR4 signalling in osteoarthritis-finding targets for candidate DMOADs. Nat Rev Rheumatol 11: 159-170, 2015.

3. Rahmati M, Mobasheri A and Mozafari M: Inflammatory mediators in osteoarthritis: A critical review of the state-of-the-art, current prospects, and future challenges. Bone 85: 81-90, 2016.

4. Barbour KE, Hootman JM, Helmick CG, Murphy LB, Theis KA, Schwartz TA, Kalsbeek WD, Renner JB and Jordan JM: Meeting physical activity guidelines and the risk of incident knee osteoarthritis: A population-based prospective cohort study. Arthritis Care Res (Hoboken) 66: 139-146, 2014.

5. Blagojevic M, Jinks C, Jeffery A and Jordan KP: Risk factors for onset of osteoarthritis of the knee in older adults: A systematic review and meta-analysis. Osteoarthritis Cartilage 18: 24-33,2010.

6. Berenbaum F and van den Berg WB: Inflammation in osteoarthritis: Changing views. Osteoarthritis Cartilage 23: 1823-1824, 2015.

7. Cifuentes DJ, Rocha LG, Silva LA, Brito AC, Rueff-Barroso CR Porto LC and Pinho RA: Decrease in oxidative stress and histological changes induced by physical exercise calibrated in rats with osteoarthritis induced by monosodium iodoacetate. Osteoarthritis Cartilage 18: 1088-1095, 2010.

8. Boudenot A, Presle N, Uzbekov R, Toumi H, Pallu S and Lespessailles E: Effect of interval-training exercise on subchondral bone in a chemically-induced osteoarthritis model. Osteoarthritis Cartilage 22: 1176-1185, 2014.

9. Siebelt M, Groen HC, Koelewijn SJ, de Blois E, Sandker M, Waarsing JH, Müller C, van Osch GJ, de Jong M and Weinans H: Increased physical activity severely induces osteoarthritic changes in knee joints with papain induced sulfate-glycosaminoglycan depleted cartilage. Arthritis Res Ther 16: R32, 2014.

10. Nam J, Perera P, Liu J, Wu LC, Rath B, Butterfield TA and Agarwal S: Transcriptome-wide gene regulation by gentle treadmill walking during the progression of monoiodoacetate-induced arthritis. Arthritis Rheum 63: 1613-1625, 2011.

11. Iijima $\mathrm{H}$, Aoyama $\mathrm{T}$, Ito $\mathrm{A}$, Yamaguchi $\mathrm{S}$, Nagai M, Tajino J, Zhang $\mathrm{X}$ and Kuroki $\mathrm{H}$ : Effects of short-term gentle treadmil walking on subchondral bone in a rat model of instability-induced osteoarthritis. Osteoarthritis Cartilage 23: 1563-1574, 2015.

12. Iijima H, Ito A, Nagai M, Tajino J, Yamaguchi S, Kiyan W, Nakahata A, Zhang J, Wang T, Aoyama T, et al: Physiologica exercise loading suppresses post-traumatic osteoarthritis progression via an increase in bone morphogenetic proteins expression in an experimental rat knee model. Osteoarthritis Cartilage 25: 964-975, 2017.

13. Galois L, Etienne S, Grossin L, Watrin-Pinzano A, Cournil-Henrionnet C, Loeuille D, Netter P, Mainard D and Gillet P: Dose-response relationship for exercise on severity of experimental osteoarthritis in rats: A pilot study. Osteoarthritis Cartilage 12: 779-786, 2004

14. Hong Y, Kim H, Lee Y, Lee S, Kim K, Jin Y, Lee SR, Chang KT and Hong Y: Salutary effects of melatonin combined with treadmill exercise on cartilage damage. J Pineal Res 57: 53-66, 2014.

15. Mizushima N: Physiological functions of autophagy. Curr Top Microbiol Immunol 335: 71-84, 2009.

16. Milner PI, Fairfax TP, Browning JA, Wilkins RJ and Gibson JS The effect of $\mathrm{O}_{2}$ tension on $\mathrm{pH}$ homeostasis in equine articular chondrocytes. Arthritis Rheum 54: 3523-3532, 2006.

17. Kim J, Kundu M, Viollet B and Guan KL: AMPK and mTOR regulate autophagy through direct phosphorylation of Ulk1. Nat Cell Biol 13: 132-141, 2011.

18. Caramés B, Taniguchi N, Otsuki S, Blanco FJ and Lotz M: Autophagy is a protective mechanism in normal cartilage, and its aging-related loss is linked with cell death and osteoarthritis. Arthritis Rheum 62: 791-801, 2010.

19. Caramés B, Taniguchi N, Seino D, Blanco FJ, D'Lima D and Lotz M: Mechanical injury suppresses autophagy regulators and pharmacologic activation of autophagy results in chondroprotection. Arthritis Rheum 64: 1182-1192, 2012.

20. López de Figueroa P,LotzMK,Blanco FJ and Caramés B: Autophagy activation and protection from mitochondrial dysfunction in human chondrocytes. Arthritis Rheumatol 67: 966-976, 2015.

21. Cetrullo S, D'Adamo S, Guidotti S, Borzì RM and Flamigni F: Hydroxytyrosol prevents chondrocyte death under oxidative stress by inducing autophagy through sirtuin 1-dependent and -independent mechanisms. Biochim Biophys Acta 1860: 1181-1191, 2016

22. Sasaki H, Takayama K, Matsushita T, Ishida K, Kubo S, Matsumoto T, Fujita N, Oka S, Kurosaka M and Kuroda R: Autophagy modulates osteoarthritis-related gene expression in human chondrocytes. Arthritis Rheum 64: 1920-1928, 2012.
23. Srinivas V, Bohensky J and Shapiro IM: Autophagy: A new phase in the maturation of growth plate chondrocytes is regulated by HIF, mTOR and AMP kinase. Cells Tissues Organs 189: 88-92, 2009.

24. Watson K and Baar K: mTOR and the health benefits of exercise Semin Cell Dev Biol 36: 130-139, 2014

25. Yang Y, Wang Y, Kong Y, Zhang X and Bai L: The effects of different frequency treadmill exercise on lipoxin A4 and articular cartilage degeneration in an experimental model of monosodium iodoacetate-induced osteoarthritis in rats. PLoS One 12: e0179162, 2017

26. Guzman RE, Evans MG, Bove S, Morenko B and Kilgore K: Mono-iodoacetate-induced histologic changes in subchondral bone and articular cartilage of rat femorotibial joints: An animal model of osteoarthritis. Toxicol Pathol 31: 619-624, 2003.

27. Pritzker KP, Gay S, Jimenez SA, Ostergaard K, Pelletier JP, Revell PA, Salter D and van den Berg WB: Osteoarthritis cartilage histopathology: Grading and staging. Osteoarthritis Cartilage 14: 13-29, 2006

28. Krenn V, Morawietz L, Burmester GR, Kinne RW, Mueller-Ladner U, Muller B and Haupl T: Synovitis score: Discrimination between chronic low-grade and high-grade synovitis. Histopathology 49: 358-364, 2006.

29. Guilak F, Alexopoulos LG, Upton ML, Youn I, Choi JB Cao L, Setton LA and Haider MA: The pericellular matrix as a transducer of biomechanical and biochemical signals in articular cartilage. Ann NY Acad Sci 1068: 498-512, 2006.

30. Livak KJ and Schmittgen TD: Analysis of relative gene expression data using real-time quantitative PCR and the 2(-Delta Delta C(T)) method. Methods 25: 402-408, 2001.

31. Roos EM and Arden NK: Strategies for the prevention of knee osteoarthritis. Nat Rev Rheumatol 12: 92-101, 2016.

32. Bomer N, Cornelis FM, Ramos YF, den Hollander W, Storms L, van der Breggen R, Lakenberg N, Slagboom PE, Meulenbelt I and Lories RJ: The effect of forced exercise on knee joints in Dio2(-/-) mice: Type II iodothyronine deiodinase-deficient mice are less prone to develop OA-like cartilage damage upon excessive mechanical stress. Ann Rheum Dis 75: 571-577, 2016.

33. Rasheed Z, Rasheed N and Al-Shaya O: Epigallocatechin-3$\mathrm{O}$-gallate modulates global microRNA expression in interleukin-1 $\beta$-stimulated human osteoarthritis chondrocytes: Potential role of EGCG on negative co-regulation of microRNA-140-3p and ADAMTS5. Eur J Nutr 57: 917-928, 2018

34. Jeon JE, Schrobback K, Meinert C, Sramek V, Hutmacher DW and Klein TJ: Effect of preculture and loading on expression of matrix molecules, matrix metalloproteinases, and cytokines by expanded osteoarthritic chondrocytes. Arthritis Rheum 65: 2356-2367, 2013

35. Wojdasiewicz P, Poniatowski $Ł A$ and Szukiewicz D: The role of inflammatory and anti-inflammatory cytokines in the pathogenesis of osteoarthritis. Mediators Inflamm 2014: 561459, 2014.

36. Barranco C: Osteoarthritis: Activate autophagy to prevent cartilage degeneration? Nat Rev Rheumatol 11: 127, 2015.

37. Caramés B, Olmer M, Kiosses WB and Lotz MK: The relationship of autophagy defects to cartilage damage during joint aging in a mouse model. Arthritis Rheumatol 67: 1568-1576, 2015.

38. Bjørkøy G,Lamark T, Brech A, Outzen H, Perander M, Overvatn A, Stenmark $\mathrm{H}$ and Johansen T: p62/SQSTM1 forms protein aggregates degraded by autophagy and has a protective effect on huntingtin-induced cell death. J Cell Biol 171: 603-614, 2005.

39. Sanchez AM, Bernardi H, Py G and Candau RB: Autophagy is essential to support skeletal muscle plasticity in response to endurance exercise. Am J Physiol Regul Integr Comp Physiol 307: R956-R969, 2014.

40. Kang R, Zeh HJ, Lotze MT and Tang D: The Beclin 1 network regulates autophagy and apoptosis. Cell Death Differ 18: 571-580, 2011.

41. Kang X, Yang W, Feng D, Jin X, Ma Z, Qian Z, Xie T, Li H, Liu J, Wang R, et al: Cartilage-specific autophagy deficiency promotes ER stress and impairs chondrogenesis in PERK-ATF4-CHOP-dependent manner. J Bone Miner Res 32: 2128-2141, 2017.

42. Khozoee B, Mafi P, Mafi R and Khan WS: Mechanical stimulation protocols of human derived cells in articular cartilage tissue engineering-a systematic review. Curr Stem Cell Res Ther 12: 260-270, 2017.

This work is licensed under a Creative Commons Attribution-NonCommercial-NoDerivatives 4.0 International (CC BY-NC-ND 4.0) License. 